\title{
Home peripheral nerve catheters: the first 24 months of experience at a children's hospital
}

\author{
Andrew Gable 1,2 \\ Candice Burrier ${ }^{1,3}$ \\ Jenna Stevens' \\ Sharon Wrona' \\ Kevin Klingele $e^{4,5}$ \\ Tarun Bhalla ${ }^{1,3}$ \\ David P Martin 1,3 \\ Giorgio Veneziano ${ }^{1,3}$ \\ Joseph D Tobias ${ }^{1,3}$ \\ 'Department of Anesthesiology and \\ Pain Medicine, Nationwide Children's \\ Hospital, Columbus, ${ }^{2}$ Heritage College \\ of Osteopathic Medicine, Ohio \\ University, Athens, ${ }^{3}$ Department of \\ Anesthesiology and Pain Medicine, \\ The Ohio State University, \\ ${ }^{4}$ Department of Orthopedics, \\ Nationwide Children's Hospital, \\ ${ }^{5}$ Department of Orthopedics, The \\ Ohio State University, Columbus, $\mathrm{OH}$, \\ USA
}

Correspondence: Candice Burrier Department of Anesthesiology and Pain Medicine, Nationwide Children's Hospital, 700 Children's Drive, Columbus, $\mathrm{OH} 43205$, USA

$\mathrm{Tel}+\mathrm{I} 6147224200$

$\mathrm{Fax}+\mathrm{I} 6147224203$

Email Candice.Burrier@

Nationwidechildrens.org

\author{
This article was published in the following Dove Press journal: \\ Journal of Pain Research \\ 18 November 2016 \\ Number of times this article has been viewed
}

Context: Home peripheral nerve catheters (PNCs) have become common practice for adult patients after major orthopedic surgery. However, use in pediatric patients is a recent application. Objectives: The purpose of this study was to review the demographics and outcomes of pediatric patients receiving a PNC at our institution.

Methods: This retrospective study included patients from October 2012 through October 2014 undergoing orthopedic procedures with a PNC placed for postoperative pain management.

Results: A total of 118 patients aged 3.2-25.3 years were identified. The types of catheters included femoral (80.5\%), interscalene (11.9\%), sciatic (5.9\%), and supraclavicular (1.7\%). The majority of patients were discharged to home on the day of surgery $(77.1 \%)$. In the postanesthetic care unit, the average pain score was 2.5 , the incidence of nausea/emesis was $5.9 \%$, and the need for opioid administration was $50.8 \%$. There were no major complications. Minor complications included a $7.6 \%$ rate of early catheter removal with $5.9 \%$ of those due to catheter leakage and an unsecure dressing. There was one case of metallic taste in the mouth without other symptoms of local anesthetic toxicity that resolved without further complication.

Conclusion: The implementation of a home PNC program in pediatric patients at our institution has been highly successful with a high rate of ambulatory catheters, low pain scores, low rates of nausea and vomiting, and no serious complications. Minor complications included leaking of the catheter and early discontinuation of the catheter.

Keywords: peripheral nerve catheter, pediatric, regional anesthesia

\section{Introduction}

Although regional anesthesia has been shown to be an effective means of providing postoperative analgesia, single shot techniques will provide only 8-12 hours of analgesia. As major orthopedic procedures may result in acute postoperative pain lasting 2-3 days, more prolonged methods of providing postoperative analgesia are needed. Home catheter programs have been shown to be safe and effective in adults. ${ }^{1}$ However, peripheral nerve catheters (PNCs) in children have had limited use and have been used primarily as an inpatient pain management therapy. Recently, there have been reports of the use of PNCs for pediatric outpatients. ${ }^{2-5}$ The Department of Anesthesiology and Pain Medicine of the Nationwide Children's Hospital launched a home catheter infusion program in October 2012. Prior to launching the program, a systematic approach was performed using the Healthcare Failure Mode and Effect Analysis to identify potential adverse effects and to develop an infrastructure to make home infusions of local anesthetics a safe and effective practice. ${ }^{6}$ Following this evaluation, a home catheter infusion program was 
initiated, and such care has become routine practice following major orthopedic surgery of the knee and shoulder. This study retrospectively reviews the initial 24 months experience with our home PNC infusion program to describe the patient and regional block demographics as well as to evaluate the efficacy, adverse effects, and technical problems.

\section{Methods}

The study was conducted at the Nationwide Children's Hospital, a tertiary children's hospital in Columbus, OH, USA. The study was reviewed by the Institutional Review Board of Nationwide Children's Hospital and deemed a quality improvement project, and therefore did not need full IRB approval, and the need for informed consent was waived. The study is a retrospective chart review of the extremity PNCs over a 24-month period from October 2012 to October 2014. The PNC patients were identified, and the data were reviewed from the Pediatric Regional Anesthesia Network (PRAN) database as well as our internal pain management database (Microsoft Access). The anesthetic records were viewed from the electronic anesthesia information system (Picis, Wakefield, MA, USA), including preoperative evaluation, peripheral block note, anesthetic record, and postanesthetic care. The inpatient records and home telephone calls were retrieved from the hospital electronic record (Epic, Verona, WI, USA).

The patient demographic information collected included patient age, weight, American Society of Anaesthesiologists' (ASA) classification, and type of surgery. Peripheral nerve block information collected included type of block, type of local anesthetic, additives to the local anesthetic solution, volume of bolus, PNC infusion local anesthetic concentration, rate of infusion, and complications. Postoperative data collected included pain scores, opioid use, nausea, emesis, postanesthetic care unit (PACU) length of stay, hospitalization, days of catheter duration, and complications. Descriptive statistics were used to analyze the data that are presented as mean \pm SD.

\section{Catheter placement technique}

The PNC was placed or supervised by an attending anesthesiologist on the pediatric regional anesthesia and acute pain management team after obtaining written informed consent. The patient was prepped with chlorhexidine, and the entire procedure was performed under strict sterile conditions. Ultrasound guidance was used for catheter placement in all cases. At our institution, we use the Braun Contiplex PNC set that includes an $18 \mathrm{G}, 5 \mathrm{~cm}$ or $10 \mathrm{~cm}$ Touhy Needle and a $20 \mathrm{G}$ polyamide closed tip catheter (B. Braun, Bethlehem, PA, USA). A bolus of local anesthetic was administered under ultrasound through the needle and then through the catheter to confirm local anesthetic spread around the nerve. The local anesthetic agent, dose, and additives were up to the discretion of the attending anesthesiologist.

PNCs in children have a high rate of dislodgement, and we have developed a technique to try to decrease this risk. The catheter was secured in place with a small drop of Dermabond $^{\mathrm{TM}}$ (Ethicon, Raleigh, NC, USA) at the skin insertion site and allowed to dry completely. The area around the catheter is prepared with mastisol, the catheter is coiled in several concentric loops, and then the catheter is secured to the skin using steri-strips. A large clear occlusive dressing of Tegaderm $^{\mathrm{TM}}$ was placed over the catheter insertion site, catheter coils, and steri-strips. The dressing was reinforced with silk tape around the edges and labeled with a "PNC" sticker.

The elastomeric home infusion device (Ambu ACTion ${ }^{\mathrm{TM}}$ Block Pain Pump) was filled with $0.1 \%$ or $0.2 \%$ ropivacaine by the hospital pharmacy (Table 5). The maximum fill volume was $400 \mathrm{~mL}$, and the pump was typically filled to $400 \mathrm{~mL}$. A member of the acute pain service set the home infusion device rate and attached it to the PNC. Prior to discharge home, the family was given instructions on how to care for the catheter, protection of the blocked limb, fall precautions, signs and symptoms of infection and local anesthetic toxicity, expectations for pain relief, emergency contact numbers, and instructions for catheter removal at home. The family was instructed to start pain medications at home when the initial block began to wear off, and they began to have some discomfort. The pain medication prescribed was up to the discretion of the attending surgeon but was typically an opioid/acetaminophen combination pill to be alternated with ibuprofen. The family was instructed to remove the catheter at the provided end time of infusion, or if the dressing became loose with the catheter exposed. They were instructed that the catheter should come out easily and to stop if there was any resistance to catheter removal. The entire catheter and pump was disposable and was discarded. A written handout with such information was provided to the family for reference. A member of the acute pain service called the family each day to answer any questions and ask about pain control and catheter complications.

\section{Results}

Over the 24-month time period, a total of 118 patients with extremity PNCs were identified. The patients ranged in age from 3 years to 25 years (15.9 \pm 3.4 years) and in weight from $11.1 \mathrm{~kg}$ to $139.5 \mathrm{~kg}(68.8 \pm 21.0)$. The American Society of Anesthesiologists' physical status was I or II in $98 \%$ of the patients and III in $2 \%$ of the patients (Table 1). Of the 118 PNCs, 95 (80.5\%) were femoral nerve catheters, 14 (11.9\%) were interscalene catheters, seven $(5.9 \%)$ were sciatic 
Table I Cohort demographics $(n=|| 8)$

Age (years)

Weight $(\mathrm{kg})$

$\operatorname{Sex}(M / F)$

ASA status I/II/III

Note: Data presented as mean \pm standard deviation (range).

Abbreviations: F, female; M, male; ASA, American Society of Anaesthesiologists'.

Table 2 Types of peripheral nerve catheters blocks $(n=118)$

\begin{tabular}{ll}
\hline Femoral & $95(80.5)$ \\
Interscalene & $14(11.9)$ \\
Sciatic & $7(5.9)$ \\
Supraclavicular & $2(1.7)$ \\
\hline
\end{tabular}

Note: Data presented as $\mathrm{n}(\%)$.

Table 3 Description of procedures $(n=\mid 18)$

\begin{tabular}{|c|c|}
\hline Procedures & n (\%) \\
\hline \multicolumn{2}{|l|}{ Knee } \\
\hline $\mathrm{ACL}$ reconstruction & $51(43.2)$ \\
\hline $\mathrm{ACL}$ reconstruction + meniscus repair & $29(24.6)$ \\
\hline$A C L$ revision & $3(2.5)$ \\
\hline Knee arthroscopy + manipulation + lysis of adhesions & $3(2.5)$ \\
\hline Knee others ${ }^{\mathrm{a}}$ & $8(6.8)$ \\
\hline \multicolumn{2}{|l|}{ Ankle/foot } \\
\hline Achilles lengthening + calcaneal lengthening & $2(1.7)$ \\
\hline $\begin{array}{l}\text { Ankle/foot others: plantar fusion, perineal nerve repair, } \\
\text { and ORIF of ankle }\end{array}$ & $3(2.5)$ \\
\hline \multicolumn{2}{|l|}{ Lower extremity } \\
\hline Below the knee amputation revision & $2(1.7)$ \\
\hline Radical resection of leg tumor & $\mathrm{I}(0.8)$ \\
\hline \multicolumn{2}{|l|}{ Shoulder } \\
\hline Bankart repair & II (9.3) \\
\hline \multicolumn{2}{|l|}{ arthroscopy + debridement } \\
\hline \multicolumn{2}{|l|}{ Upper extremity } \\
\hline Below elbow amputation & $2(1.7)$ \\
\hline Arm others: ORIF humerus & $\mathrm{I}(0.8)$ \\
\hline
\end{tabular}

Note: aDeep hardware removal + lysis of adhesions, medial patellofemoral ligament reconstruction with allograft, ORIF of patella, ORIF of tibial tubercle, ORIF of knee, ORIF of tibial spine + meniscus repair, extensor realignment, and knee arthroscopy + tibial tubercle transfer.

Abbreviations: $A C L$, anterior cruciate ligament; ORIF, open reduction and internal fixation.

catheters, and two $(1.7 \%)$ were supraclavicular catheters (Table 2). The types of surgical procedures performed are listed in Table 3. The most common procedures for extremity PNCs were anterior cruciate ligament (ACL) reconstruction (43.2\%), ACL reconstruction + meniscus repair (24.6\%), and Bankart repair of the shoulder (9.3\%).

All of the PNCs were placed under ultrasound guidance, and all but one was performed under general anesthesia, including the 14 interscalene blocks. One catheter was placed under sedation. The local anesthetic agent, ropivacaine, was used in all except one patient, in whom $0.5 \%$ bupivacaine was used. The concentration of ropivacaine was $0.2 \%$ in five patients ( $4.2 \%), 0.3 \%$ in one patient $(0.8 \%)$, and $0.5 \%$ in 111 patients (94\%). Epinephrine was used as a test dose to detect intravascular injection in 89 patients $(75.4 \%)$, and dexamethasone was used as an additive to prolong the initial block in 85 patients (72\%) (Table 4). The initial block is typically a denser block with a higher concentration of local anesthetic, and the dexamethasone is given to prolong the duration of the higher density block.

Postoperatively, the PNC was connected to a continuous infusion of ropivacaine. The concentration of ropivacaine was $0.1 \%$ in $3.4 \%$ of the patients and $0.2 \%$ in $96.6 \%$ of the patients. The average infusion rate was $7.6 \pm 1.2 \mathrm{~mL} / \mathrm{h}$ (range: $5.0-10.0 \mathrm{~mL} / \mathrm{h})$. The catheter was left in place for an average of $2.0 \pm 0.2$ days (range: $0-4$ days). One $(0.8 \%)$ catheter stayed in place for 0 days, eight $(6.7 \%)$ catheters stayed for 1 day, 86 (72.9\%) catheters stayed for 2 days, 21 (17.8\%) catheters stayed for 3 days, and two (1.7\%) catheters stayed for 4 days (Table 5 ). The reason for catheter removal was that the local anesthetic infusion was complete in 109 (92.4\%) patients. In seven patients (5.9\%), the catheter was removed early due to catheter leakage with an unsecured dressing and

Table 4 Peripheral nerve block catheter placement information $(n=118)$

\begin{tabular}{ll}
\hline Ultrasound guidance & II (I00\%) \\
Ropivacaine bolus concentration $(0.2 \% / 0.3 \% / 0.5 \%)^{\mathrm{a}}$ & $5 / \mathrm{I} / \mathrm{II} \mathrm{I}$ \\
Local anesthetic additives & \\
I:200,000 epinephrine & $89(75.4 \%)$ \\
Dexamethasone & $85(72.0 \%)$ \\
\hline Note: ${ }^{\circ}$ One patient received bupivacaine. &
\end{tabular}

Table 5 Peripheral nerve block catheter infusion information $(n=118)$

\begin{tabular}{ll}
\hline Ropivacaine infusion concentration $(0.1 \% / 0.2 \%)$ & $4 / 114$ \\
Ropivacaine infusion rate $(\mathrm{mL} / \mathrm{h})$ & $7.6 \pm 1.2(5.0-10.0)$ \\
Catheter duration $(0 / 1 / 2 / 3 / 4$ days $)$ & $1 / 8 / 86 / 2 \mathrm{I} / 2$ \\
\hline
\end{tabular}

Note: Data presented as mean \pm standard deviation (range).

Table 6 Reason for catheter removal $(n=118)$

\begin{tabular}{ll}
\hline Infusion complete & $109(92.4)$ \\
Unsecure dressing due to leaking of catheter & $7(5.9)$ \\
Inadvertent early removal (fell out) & $2(1.7)$ \\
\hline
\end{tabular}

Note: Data presented as $\mathrm{n}(\%)$.

Table 7 Peripheral nerve block catheter complications $(n=118)$

\begin{tabular}{ll}
\hline Early removal & $9(7.6)$ \\
Local anesthetic toxicity & $\mathrm{I}^{\mathrm{a}}(0.8)$ \\
Vascular puncture/bleeding & $0(0)$ \\
Infection & $0(0)$ \\
Nerve injury & $0(0)$ \\
Catheter difficult to remove & $0(0)$ \\
ED visit for uncontrolled pain & $0(0)$ \\
\hline
\end{tabular}

Notes: Data presented as $n$ (\%). 'Local anesthetic toxicity unlikely. See text body for explanation.

Abbreviation: ED, emergency department. 
catheter exposure. In two patients $(1.7 \%)$, the catheter was inadvertently removed (Table 6). All patients were able to remove the catheters without difficulty at home (Table 7).

There were no major complications from PNCs. Minor complications from the PNC included early removal in $7.6 \%$ of catheters and one case of metallic taste with possible local anesthetic toxicity. There were no complications related to infection, bleeding, or nerve injury. None of the PNC patients were seen in the emergency department for uncontrolled pain or other concerns. There were no readmissions for pain (Table 7).

The patient with the reported metallic taste was a 20 -yearold male weighing $87.1 \mathrm{~kg}$ who underwent a posterior Bankart repair with an uncomplicated ultrasound-guided interscalene nerve block. The block was performed under general anesthesia with $25 \mathrm{~mL}$ of $0.5 \%$ ropivacaine $(1.44 \mathrm{mg} / \mathrm{kg})$ with $1: 200,000$ epinephrine. While in the PACU, the patient complained of a metallic taste in his mouth but was awake and alert, denied blurred vision, tinnitus, and was otherwise feeling well. Although the likelihood of local anesthetic toxicity was low, the local anesthetic infusion was clamped. The patient was observed without any further symptoms and was discharged to home with the local anesthetic infusion clamped. The family was given instruction to unclamp the infusion when the patient woke up the next morning or when he began to feel the initial block wear off as long as no other problems arose. They were given instructions to reclamp the local anesthetic infusion if any symptoms of local anesthetic developed. Later that evening, the block began to wear off, the metallic taste had resolved, and the infusion of local anesthetic was unclamped. The patient experienced good pain relief and did not have any additional symptoms of local anesthetic toxicity.

Postoperatively, the average length of PACU stay was $66 \pm 37$ minutes (range: 18-266 minutes). There was a 5.9\% incidence of nausea and/or emesis. Sixty (50.8\%) patients required a dose of opioid in the PACU. At our institution, it is a standard practice to record postoperative Visual Analogue Scale pain scores every 15 minutes during recovery from anesthesia. Pain scores 1-4 are the first through fourth pain scores recorded, scores 5-8 are the fifth through eighth pain scores recorded, and scores 9-12 are the ninth through twelfth pain scores recorded in the PACU. The average of pain scores 1-4 was 2.5 , that of pain scores 5-8 was 3.1 , and that of pain scores 9-12 was 3.6 (Table 8).

In our cohort, $91(77.1 \%)$ patients were discharged to home on the day of surgery. Of the 27 patients admitted to the hospital, nine patients were admitted as a result of uncontrolled pain (Table 8), seven patients were admitted for
Table 8 Postoperative outcomes $(n=\mid 18)$

\begin{tabular}{llll}
\hline Length of PACU stay (minutes) & $66 \pm 37($ (18-266) & \\
Incidence of nausea/emesis & $7(5.9)$ & \\
Need for opioid administration in PACU & $60(50.8)$ & \\
Hospital admission & $27(22.9)$ & \\
Hospital admission for uncontrolled pain & $9(7.6)$ & & \\
& $1-4$ & $5-8$ & $9-12$ \\
Average pain scores recorded $^{\mathrm{a}}$ & 2.5 & 3.1 & 3.6 \\
\hline
\end{tabular}

Notes: Data presented as mean \pm standard deviation (range) or $\mathrm{n}(\%)$. ${ }^{\text {PPain }}$ scores $1-4$ are the first to fourth pain scores recorded, scores 5-8 are the fifth to eighth pain scores recorded, scores 9-12 are the ninth to twelfth pain scores recorded. Pain scores are recorded approximately every 15 minutes.

Abbreviation: PACU, postanesthetic care unit.

neurovascular monitoring, six patients required admission per standard protocol, three patients were admitted for overnight observation, and two patients were admitted at the request of our orthopedic surgeons. Of the patients admitted for poor pain control, five patients had an ACL reconstruction, two patients had knee manipulation with the lysis of adhesions, one patient had a plantar fusion, and one patient had an ORIF of the humerus.

\section{Discussion}

Our data demonstrate that ambulatory PNCs can be safely used in the pediatric population after a systematic and careful approach to implementation of a pediatric PNC program. The steps we used to ensure safety were development of infrastructure, careful patient selection, and close patient follow-up. Using the HMFEA system, we identified potential safety issues and created an infrastructure for the PNC program. ${ }^{6}$ The program development included education for all members of the health care team involved in the PNC program, standard order sets in the EMR, development of hospital policies and procedures, an educational handout for the families, and a 24-hour pager for emergency phone calls to the acute pain service from parents. Furthermore, we used careful patient selection for the procedures. Families had to be comfortable with caring for a catheter at home and had to be English speaking so that we could clearly and quickly communicate over the telephone in case of an emergency. We reserved ambulatory PNCs for relatively healthy patients. In our study, all but two patients had an ASA classification status of I or II. The two patients with an ASA classification of III were cancer patients undergoing amputation procedures and were admitted for postoperative observation. The average age of our patients was 15.9 years, and the average weight was $68.8 \mathrm{~kg}$ with the majority being adolescents. Our two youngest patients (ages 3 years and 5 years) were admitted for overnight observation. Finally, we had close follow-up of all of our patients. Prior to discharge, the family was given detailed written and verbal instructions 
about the PNC. The patients were followed on a daily basis by the acute pain service, and the family had a pager number to call in the case of an emergency.

In our study, we had very few complications, and all of them were minor. The most common complication was catheter leakage causing the Tegaderm ${ }^{\mathrm{TM}}$ dressing to become loose and nonocclusive. Due to the concern of infection with a potentially exposed catheter, it is our practice to remove the catheter in this situation. In our cohort of 118 patients, seven $(5.9 \%)$ patients had catheter leakage and early removal of the catheter. In two other patients, the catheter fell out without mention of catheter leakage for a total early catheter removal rate of $7.6 \%$. This result is consistent with the literature. Ganesh et $\mathrm{al}^{2}$ experienced leakage in five $(2.3 \%)$ patients, and this was the cause of early removal in one of them. Other studies have demonstrated accidental catheter removal in $15 \%{ }^{7}$ and $10.5 \%{ }^{8}$ of patients. To help decrease the incidence of catheter leakage, we typically occlude the catheter insertion site with a small drop of Dermabond ${ }^{\mathrm{TM}}$ to seal the site. Another method to decrease catheter leakage recently reported in the literature is the use of catheter over needle PNC systems. ${ }^{9}$ In the catheter over needle system, the catheter insertion site does not leave a potential space around the catheter as a path of infusion leakage. As the most common complication in our cohort, steps to decrease catheter leakage and early dislodgement would make a clinically significant improvement. Since the collection of this data, we have initiated the catheter over needle system for most of our PNCs.

We had one case of potential local anesthetic toxicity. Systemic toxicity of local anesthetics can occur after administration of an excessive dose, with rapid absorption, or because of an accidental intravenous injection. ${ }^{10}$ Early signs of neurologic toxicity can include metallic taste, tinnitus, and malaise. ${ }^{11}$ Our one patient with a metallic taste in his mouth in the PACU received a total of $125 \mathrm{mg}(1.44 \mathrm{mg} / \mathrm{kg})$ of ropivacaine in an interscalene block, which is significantly less than the maximum dose limit. He did not experience any other symptoms of local anesthetic toxicity, and we were able to continue the infusion without return of symptoms of local anesthetic toxicity. We decided to continue the infusion due to the low likelihood of local anesthetic toxicity in this patient. Ganesh et $\mathrm{al}^{2}$ experienced a similar complication, with a patient who complained of tinnitus 24 hours after an interscalene catheter placement that resolved with clamping of the catheter. In their patient, the catheter was removed. As per Krane and Polaner, ${ }^{12}$ it is impossible to determine whether these examples truly represented toxicity or, perhaps, a placebo response in children who may have been told to be on alert for symptoms of local anesthetic toxicity.
We did not see any complications of vascular puncture, positive test dose, local anesthetic toxicity with neurologic or cardiovascular compromise, vascular puncture/hematoma, nerve injury, infection, catheters that were difficult to remove, or emergency room visits/readmissions for uncontrolled pain. Our university is part of the PRAN, and as part of the network, complications were collected prospectively, which would help increase reporting of such events. However, some data may be underreported or missed. Furthermore, rare complications may not be captured in our sample size.

One of the major advantages of improved analgesia with PNC catheters is the ability to discharge patients home following major orthopedic procedures. Prior to the initiation of our home PNC program, it was standard practice to admit all patients undergoing ACL reconstructions and Bankart repairs overnight to the hospital for pain control. In our review, $91(77.1 \%)$ patients were discharged to home on the day of surgery.

While our study was retrospective and we cannot demonstrate superiority of pain control with the PNC technique over other techniques, our patients had excellent postoperative outcomes, including high rates of same day discharge, low pain scores, low rates of PACU opioid administration, and low rates of postoperative nausea or vomiting. We have previously published our data demonstrating a reduction in hospital admission rate after implementation of our PNC program, reducing the hospital admission rate for knee surgery from $95 \%$ in 2010 to $2.5 \%$ in $2013 .{ }^{13}$ In the current study, $91(77.1 \%)$ patients were discharged to home on the day of surgery; that is, comparable to the results of Visoiu et $\mathrm{al}^{5}$ with a $76.7 \%$ same day of surgery discharge rate, but higher than the same day discharge rate reported by other groups, including $50 \%$ by Ganesh et al, ${ }^{2} 35.6 \%$ by Gurnaney et al, ${ }^{4}$ and $4.2 \%$ by Ludot et al. ${ }^{14}$ As a result of our high success rate with discharge to home with the PNCs, many of these procedures are now occurring at our outpatient surgery center.

Our postoperative pain scores were excellent with the averages for the first four pain scores, fifth through eighth pain scores, and ninth through twelve pain scores being 2.5 , 3.1 , and 3.6, respectively. The postoperative goal on our acute pain service is to have a VAS pain score of $\leq 4$, which typically correlates with pain that is tolerable and does not require intervention. The average pain scores in our cohort were well below this and demonstrate excellent pain control. While recovering in PACU, 60 (50.8\%) patients required a rescue opioid administration, which is comparable to the studies by Gurnaney et $\mathrm{al}^{4}{ }^{4}$ Ganesh et $\mathrm{al}^{2}{ }^{2}$ and Visoiu et $\mathrm{al}^{5}$ with $87.3 \%, 35 \%$, and $62.6 \%$ of patient's requiring opioid administration, respectively. Our PACU length of stay aver- 
aged 66 minutes with a wide range of times (18-266). One reason for the variation was likely due to the availability of the acute pain service to connect the PNC to the pump and complete the family teaching prior to discharge. We now allow the patients to proceed to Phase II recovery when PACU discharge criteria are met and the acute pain service will complete the teaching and start the infusion in Phase II recovery. Postoperative nausea or vomiting was rare in our study, with only seven (5.9\%) patients experiencing nausea and/or emesis. Nausea and emesis was reported in $24.8 \%$ of patients by Visoiu et al, ${ }^{5}$ in $14 \%$ of patients by Ganesh et al, ${ }^{2}$ in $14.6 \%$ of patients by Dadure et al, ${ }^{11}$ and in $15 \%$ of patients by Paut et al. ${ }^{15}$ Our rates of nausea and/or emesis may have been underreported due to the retrospective nature of the data.

The major limitation of this study is that most of the data were collected retrospectively. However, the details of the initial block, catheter infusion and complications were collected prospectively as part of the PRAN database. Another limitation is that we did not have a standardized interview for the postoperative phone call to patients with PNCs and we, therefore, did not collect quantifiable ambulatory pain scores, nausea incidence, or patient satisfaction scores. Another limitation is that we did not have a control group. We considered a historical control; however, we did not have postoperative pain scores for the ambulatory catheters. Another limitation of the study is that we did not control the technique of the PNCs or the postoperative pain regimen. We have many different anesthesiologists placing the PNCs with varying preferences and techniques. In the future, it would be helpful to have prospective randomized controlled studies to evaluate the efficacy of $\mathrm{PNC}$ in children as compared to standard pain management.

\section{Conclusion}

We report the successful implementation of an outpatient pediatric PNC program with a high rate of ambulatory catheters, good pain control, and no major complications. This date provides further evidence for the safety and efficacy of pediatric PNCs in the outpatient setting.

\section{Disclosure}

The authors report no conflicts of interest in this work.

\section{References}

1. Ilfeld BM. Continuous peripheral nerve blocks: a review of the published evidence. Anesth Analg. 2011;113(4):904-925.

2. Ganesh A, Rose JB, Wells L, et al. Continuous peripheral nerve blockade for inpatient and outpatient postoperative analgesia in children. Anesth Analg. 2007;105(5):1234-1242.

3. Ilfeld BM, Smith DW, Enneking FK. Continuous regional analgesia following ambulatory pediatric orthopedic surgery. Am J Orthop. 2004;33:405-408.

4. Gurnaney H, Kraemer W, Maxwell L, Muhly W, Schleelein L, Ganesh A. Ambulatory continuous peripheral nerve blocks in children and adolescents: a longitudinal 8-year single center study. Anesth Analg. 2014;118(3):621-627.

5. Visoiu M, Joy LN, Gudziak JS, Chelly JE. The effectiveness of ambulatory continuous peripheral nerve blocks for postoperative pain management in children and adolescents. Pediatr Anesth. 2014;24(11): $1141-1148$.

6. Bhalla T, Dairo OO, Martin DP, et al. A proactive risk assessment by utilizing healthcare failure mode and effect analysis (HFMEA) for safe implementation of peripheral nerve catheters in pediatric patients. Anaesth Pain Intensive Care. 2014;18:21-24.

7. Dadure C, Bringuier S, Raux O, et al. Continuous peripheral nerve blocks for postoperative analgesia in children: feasibility and side effects in a cohort study of 339 catheters. Can JAnesth. 2009;56(11):843-850.

8. Capdevila X, Pirat P, Bringuier S, et al. Continuous peripheral nerve blocks in hospital wards after orthopedic surgery. Anesthesiology. 2005;103:1035-1045.

9. Tsui BC, Ip VH. Catheter-over-needle method reduces risk of perineural catheter dislocation. Br J Anaesth. 2014;112(4):759-760.

10. Dewaele S, Santos AC. Toxicity of local anesthetics; 2013. Available from: http://www.nysora.com/regional-anesthesia/foundations-ofra/3075-toxicity-of-local-anesthetics.html. Accessed August 11, 2016.

11. Dadure C, Capdevila X. Peripheral catheter techniques. Pediatr Anesth. 2012;22:93-101.

12. Krane EJ, Polaner D. The safety and effectiveness of continuous peripheral nerve blockade in children. Anesth Analg. 2014;118:499-500.

13. Schloss B, Bhalla T, Klingele K, Phillips D, Prestwich B, Tobias JD. A retrospective review of femoral nerve block for postoperative analgesia after knee surgery in the pediatric population. J Pediatr Orthop. 2014;34(4):459-461.

14. Ludot H, Berger J, Pichenot V, Belouadah M, Madi K, Malinovsky JM. Continuous peripheral nerve block for postoperative pain control at home: a prospective feasibility study in children. Reg Anesth Pain Med. 2008;33(1):52-56.

15. Paut O, Sallabery M, Schreiber-Deturmeny E, Rémond C, Bruguerolle B, Camboulives J. Continuous fascia iliaca compartment block in children: a prospective evaluation of plasma bupivacaine concentrations, pain scores and side effects. Anesth Analg. 2001;92(5):1159-1163.
Journal of Pain Research

Publish your work in this journal

The Journal of Pain Research is an international, peer reviewed, open access, online journal that welcomes laboratory and clinical findings in the fields of pain research and the prevention and management of pain. Original research, reviews, symposium reports, hypothesis formation and commentaries are all considered for publication.

\section{Dovepress}

The manuscript management system is completely online and includes a very quick and fair peer-review system, which is all easy to use. Visit http://www.dovepress.com/testimonials.php to read real quotes from published authors. 